\title{
Consideraciones de acercamiento iniciales para la comprensión de la importancia de la estrategia de la Política Monetaria en Costa Rica
}

\author{
Elizabeth Quirós García \\ Escuela de Lenguas Modernas \\ Universidad de Costa Rica \\ elizabeth.quiros@ucr.ac.cr
}

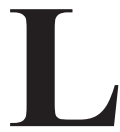
a política monetaria es un instrumento de la política económica cuyo objetivo principal es incidir sobre variables macroeconómicas como la inflación, el empleo, el crecimiento económico y los tipos de cambio. Se esperaría que la política económica de una nación pueda incidir sobre la volatilidad de las variables macroeconómicas para poder favorecer el crecimiento sostenido a largo plazo. La manera en la cual la política monetaria pretende influir sobre las variables mencionadas es por medio de los tipos de interés y la oferta monetaria, que en términos sencillos se refiere a la emisión monetaria o impresión de dinero. Es así como, y de acuerdo con Cuadrado (1995), citado por León y de la Rosa (2005):

la política monetaria consiste en la acción consciente emprendida por las autoridades monetarias, o la inacción deliberada, para cambiar la cantidad, la disponibilidad o el coste del dinero, con objeto de contribuir a lograr algunos de los objetivos básicos de la política económica. El más importante para la política monetaria es, sin duda, la estabilidad de precios, pero también puede cooperar en el logro de un crecimiento sostenido y, obviamente, a favor del equilibrio externo.

(...) la política monetaria va a influir en el comportamiento de las variables claves del sistema económico, como la producción, el empleo y los precios; además, también va a incidir en la Balanza de $\mathrm{Pa}$ gos. Por otra parte, como es conocido en la literatura macroeconómica, la generación de crecimiento económico y de empleo implica un costo en términos de que provoca presiones inflacionarias. (4)

\section{Agregados monetarios (definición y clasificación)}

Los agregados monetarios pueden definirse como la suma del dinero en circulación dentro de una economía más el saldo vivo de determinados pasivos de las instituciones financieras que tienen un alto grado de 
liquidez. Se espera que los líderes de un país sepan qué lineamientos deben seguirse en cuanto al dinero y su disponibilidad de manera que su uso sea más o menos dinámico, siempre considerando fortalecer la economía de la nación. (Suchar 24 de agosto)

Los agregados monetarios más utilizados se constituyen a partir de la emisión, la base monetaria y la cantidad de dinero en circulación (que se mide a partir de las diferentes clasificaciones):

1. Emisión monetaria: está constituida a partir de los billetes y monedas, emitidos y puestos en circulación, por el Banco Central de Costa Rica.

2. Base monetaria: esta se define a partir de la creación primaria del dinero, representando un pasivo del Banco Central, mediante el cual la autoridad monetaria puede influir en la liquidez de la economía.

En cuanto a los agregados monetarios, los cuales son "segregados de acuerdo a la liquidez del activo monetario que representan" (Suchar 24 de agosto) y de acuerdo con Díaz-Giménez, los niveles/categorización establecida de estos y son:

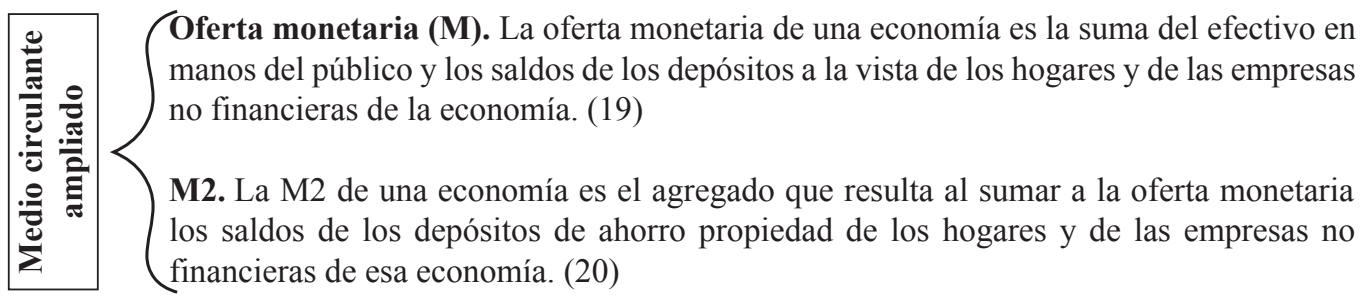

\footnotetext{
इ M3. La M3 de una economía es el agregado que resulta al sumar a la M2 los saldos de los depósitos a plazo propiedad de los hogares y de las empresas no financieras de esa economía. (20)

M4. La M4 en una economía representará la riqueza financiera total.
}

La liquidez total representa todo el dinero en efectivo "que maneja la población y cuyo origen debe de ser local" (Suchar 24 de agosto). Un dato significativo se desprende del hecho de que en una economía el dinero debe "trabajar", es decir, este debe producir.

\section{Banco Central y liquidez monetaria}

El Banco Central es la principal autoridad monetaria, cambiaria y crediticia y tiene a su cargo, en exclusiva, la ejecución de la política monetaria por medio de las herramientas de esta tales como las tasas de interés y la oferta monetaria o el flujo de dinero que llega a la economía. Esta autoridad tiene a su cargo esta política pero también la supervisión y la estabilidad del sistema financiero en su conjunto.

De acuerdo con la Superintendencia General de entidades Financieras (SUGEF), 
(2002) el Banco Central de Costa Rica tiene tres instrumentos para controlar la oferta monetaria:

1. Las operaciones de mercado abierto: compraventa de títulos valores por parte de la autoridad monetaria.

2. Las reservas exigidas mediante el encaje mínimo legal.

3. El redescuento y los préstamos de emergencia: créditos a plazo con tasas penalizadas. (11)

Por otra parte, es importante mencionar que las condiciones para lograr un crecimiento económico sostenido, a largo plazo, las persigue el Banco Central con la utilización de los instrumentos de política monetaria y es entonces cuando, a partir de estos, se gradúa tanto la cantidad de dinero (oferta monetaria) que fluye hacia la economía como el precio dinero (tipos de interés) que llega a esta.

Existen dos tipos de política monetaria: una contractiva y expansiva. Cuando el Banco Central detecta que la economía se ha acelerado excesivamente, se utiliza la política contractiva la que se caracteriza por aumentar los tipos de interés y se reduce la oferta monetaria o impresión de dinero. El objetivo principal de este tipo de política monetaria es la desaceleración de la economía y aun cuando esta meta pudiera ser objetable, es necesaria en el momento en que se detecte que el crecimiento económico es excesivamente rápido y nazca a partir de la especulación en mercados financieros o inmobiliarios, por ejemplo. Consecuentemente, y de acuerdo con Heredia Sánchez, se generará "una disminución de la oferta monetaria, produciendo, en este caso, un aumento del tipo de interés, lo que reduce la inversión privada y por tanto el nivel de producción y empleo". (5)

Por el contrario, cuando la economía se encuentra en recesión, se considerará una política monetaria expansiva, en este caso, se reducen los tipos de interés de referencia y aumentan los flujos de dinero hacia la economía real. Así se busca que haya un mayor crecimiento económico y/o se reducen las consecuencias adversas de la recesión, especialmente al influir sobre la producción, la inflación y el empleo de la nación. Según Heredia Sánchez,

Cuando el Banco Central lleva a cabo una política monetaria expansiva; se produce un incremento de la oferta monetaria. Este tipo de políticas producen, además, un efecto expansivo sobre el nivel de producción y empleo, ya que, como veremos, provocan una disminución del tipo de interés del mercado favoreciendo la inversión privada $\mathrm{y}$ consecuentemente el nivel de producción y empleo. (5)

Es así como existe una estrecha relación entre la cantidad de dinero en circulación y el nivel de precios de una economía que conlleva a que los bancos centrales puedan incidir sobre los precios de los productos y la inflación y su control, todo a partir de la intervención que se tenga de la emisión de dinero.

\section{Relación entre la inflación y los agrega- dos monetarios}

La inflación se define como el aumento generalizado y sostenido en el nivel de precios en la economía que inciden en la capacidad 
de compra del dinero. Al ser Costa Rica un país emergente y no desarrollado el cálculo de la inflación se realiza a partir de los productos de la canasta básica; en economías desarrolladas se utilizan productos durables. Cuando se enfrenta la inflación, la presión en los precios de los productos básicos influirá en los precios generales de la economía, ocasionando así que la inflación total aumente y reduciendo de esta manera el poder de compra del dinero.

En una economía, el control de la inflación es uno de los objetivos más importantes del Banco Central. De acuerdo con Heredia Sánchez, la "estrecha relación existente entre la cantidad de dinero en circulación y el nivel de precios de una economía permite que el Banco Central pueda incidir sobre la inflación a partir del control de la emisión monetaria" (2). Consiguientemente, se esperaría que el Banco Central utilice sus instrumentos para el bienestar de la población de un país.

Cabe aclarar que el Banco Central no tiene un control directo sobre los precios de mercado de los bienes o servicios en tanto son determinados por medio de la interacción de la oferta y la demanda. Sin embargo, lo que es un hecho es que la política monetaria puede influir sobre el proceso de establecimiento de los precios y de esta forma impactar de manera indirecta en la meta de inflación de una nación.

Es así como puede presumirse que el Banco Central debe estar atento al dinamismo del mercado así como estar consciente de los efectos que su política conlleva en las transacciones diarias de las personas, la determinación de precios y la economía en el nivel macro. Es entonces cuando el tema de agregados monetarios cobra relevancia ya que el Banco Central conducirá su política monetaria de manera tal que afecte aquellos escenarios bajo los cuales se satisfacen las necesidades de liquidez en la economía.

Por ejemplo, si parte de los objetivos de la política monetaria es incidir sobre los tipos de cambio y tasas de interés y estas últimas van en disminución, el circulante tendría una tendencia a aumentar (política expansiva), hecho que a su vez podría generar una dinamización de la inversión privada en la economía (personas físicas y jurídicas). Consecuentemente, este hecho puede derivar en un aumento del consumo que a su vez puede generar un incremento en la inflación; si esto sucediera, el Banco Central podría intervenir con una política restrictiva, disminuyendo el circulante para tratar así, con estrategias de política monetaria, de controlar la inflación.

\section{El crédito en Costa Rica: 2014-2015}

Previo a la discusión del tema del crédito en los años 2014 y 2015, es fundamental destacar que en el país, el aparato productivo está mayormente representado por el sector privado de la economía. Si bien es cierto que el sector público genera inversión, está enfocada principalmente en infraestructura, educación, salud y gastos administrativos. Es así como, en el informe La economía costarricense en el 2015 y perspectivas 2016-2017, se presentan los siguientes datos con respecto al crédito en el sector privado: 


\section{Crédito al sector privado}

\begin{tabular}{|l|c|c|c|}
\hline & $\begin{array}{c}\text { Moneda } \\
\text { Nacional }\end{array}$ & $\begin{array}{c}\text { Moneda } \\
\text { Extranjera }\end{array}$ & Total \\
\hline $\mathbf{2 0 1 4}$ & & & \\
\hline I Semestre & 15,2 & 13,9 & 14,6 \\
\hline II Semestre & 19,6 & 8,9 & 14,9 \\
\hline Anual & 17,4 & 11,4 & 14,8 \\
\hline $\mathbf{2 0 1 5}$ & & & \\
\hline I Semestre & 9,1 & 9,1 & 12,8 \\
\hline II Semestre & 13,3 & 13,3 & 12,1 \\
\hline Anual & 11,2 & 11,2 & 12,4 \\
\hline
\end{tabular}

Tabla 1, BCCR La economía costarricense en el 2015 y perspectivas 2016-2017

Tal y como puede observarse, durante el año 2014, el crédito al sector privado en moneda nacional presentó un aumento del primer semestre $(15,2)$ al segundo $(19,6)$; sin embargo, para el primer semestre del año 2015 tuvo una reducción del 6,1. La variación 2014-2015 alcanzó un 11,2. Por su parte, el crédito en moneda extranjera mantuvo una variación anual similar - de 11,4 a 11,2. Puede afirmarse, entonces, que aun cuando el crédito en moneda nacional es mayor que el crédito en moneda extranjera, este último se mantiene a pesar de que la economía costarricense no está dolarizada.

Sergio Morales Chavarría, en su artículo " $78 \%$ de los créditos en dólares pertenecen a deudores que ganan en colones", afirma que:

la mayoría de los préstamos en dólares en Costa Rica son adeudados por personas y empresas cuyos ingresos principales están en colones. Lo anterior implica que estos deudores tienen a cuestas el riesgo cambiario, es decir, las variaciones en que el tipo de cambio les puede llegar a afectar su capacidad de pago.

De acuerdo con Olivier Castro, presidente del Banco Central de Costa Rica, y Róger Morales, quien es el director económico de esta misma institución y citados por Morales, ellos afirman estar preocupados por "el aumento de la dolarización de la economía y el incremento en el crédito en moneda extranjera a clientes no generadores... Un choque en el tipo de cambio y el incremento en los niveles de mora pone en peligro algunos bancos y la estabilidad del sistema."

Para muchos, el hecho de que las tasas de interés en moneda extranjera sean más bajas es una razón para solicitar créditos; sin embargo, como lo establecen las autoridades bancarias, si los solicitantes generan en moneda nacional, la situación se vuelve muy riesgosa, como puede apreciarse en el siguiente gráfico:

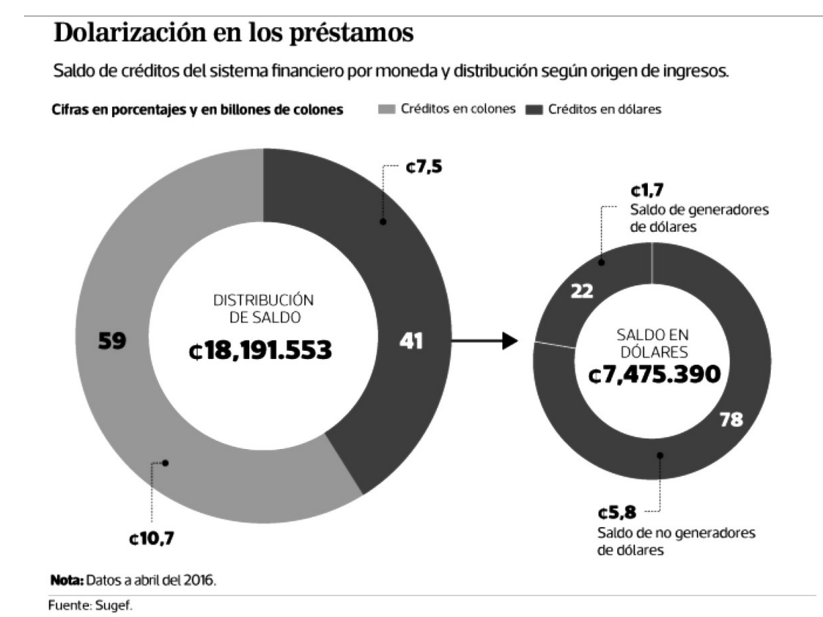

Gráfico 1: Fuente El Financiero "78\% de los créditos en dólares pertenecen a deudores que ganan en colones", 28 de junio del 2016 


\section{Beneficios de una buena estrategia de política monetaria}

De acuerdo con las decisiones en cuanto a la política monetaria en una economía, estas pueden impactar de manera negativa o positiva a los habitantes de una nación. La base monetaria y el tipo de interés van a impactar directamente la cantidad de dinero existente, el crédito bancario y el tipo de interés a largo plazo así como el tipo de cambio, los que a su vez repercutirán en dos variables macroeconómicas fundamentales: el empleo y la inflación. Este pareciera ser un juego de dominó, en donde una pieza impacta no solo a la adyacente sino que va más allá de esta hasta generar una reacción (efecto) en el conjunto total.

Es fundamental tener presente que para un país, las variables de inflación así como el desempleo deberían ser prioritarias para tratar no de eliminar la pobreza, lo cual es casi imposible, sino la desigualdad. El concepto de pobreza ha sido definido, analizado y discutido en los diferentes estadios de conocimiento de la historia humana. Como lo establece Macías (2014), "la pobreza no es algo nuevo, no es un fenómeno exclusivo de la historia reciente. Lo que es nuevo es la sospecha cada vez más evidente de que el crecimiento económico por sí mismo no puede resolver el problema, ni siquiera aliviarlo en un tiempo razonable" (1). Si esta premisa es cierta, entonces se necesita que la política monetaria de un país sea estratégica para poder impactar positivamente a su población, al estabilizar la inflación y reducir el índice de desempleo.

\section{Referencias}

Banco Central de Costa Rica (2016). La Economía costarricense en el 2015 y perspectivas para el 2016-2017. San José.

Banco Central de Costa Rica (2016). Revisión Programa Macroeconómico 2016-2017. San José.

Díaz-Giménez, J. (2010). El Dinero y los Agregados Monetarios. (sin fuente)

Garcés Díaz, D. (2003). Agregados monetarios, inflación y actividad económica en México. Estudios Económicos, enero-junio, 37-78.

Heredia Sánchez, A. (2015). Aproximación a la confección de los panoramas y el balance monetario: algunas experiencias internacionales. Revista Cubana de Economía Internacional, número 3, 77-93.

León León, J. y de la Rosa Mendoza J. R. (2005). Política monetaria, cortos y estabilidad macroeconómica Análisis Económico, 291-326.

Macías Vázquez, A. (2014). Crecimiento, desigualdad y pobreza: estado de la cuestión. Revista de Economía Institucional, 101-126.

Mankiw,N.G.(2010). Principles of Microeconomics. USA: Mc-Graw-Hill.

. (2012). Principios de Economía. México: McGraw-Hill.

Morales Chavarría, S. (2016). 78\% de los créditos en dólares pertenecen a deudores que ganan en colones. $E l$ Financiero, Costa Rica, 28 de junio. 
Schiller, R. (2012). Monetary Policy, Yale Courses. [Archivo de video]. http: https://www.youtube.com/ watch?v=_SpIaGTq0u8

Suchar Zomer, D. Teoría y Política Monetaria. Universidad Latina de Costa Rica. 24 de agosto de 2016.

SUGEF (2002). Encaje Mínimo Legal. San José: PPT. 
